\title{
Strong convergence of a self-adaptive method for the split feasibility problem
}

\author{
Yonghong Yao ${ }^{1}$, Mihai Postolache ${ }^{2 *}$ and Yeong-Cheng Liou ${ }^{3}$
}

\author{
${ }^{*}$ Correspondence: \\ mihai@mathem.pub.ro \\ ${ }^{2}$ Faculty of Applied Sciences, \\ University 'Politehnica' of Bucharest, \\ Splaiul Independentei 313, \\ Bucharest, 060042, Romania \\ Full list of author information is \\ available at the end of the article
}

\begin{abstract}
Self-adaptive methods which permit step-sizes being selected self-adaptively are effective methods for solving some important problems, e.g., variational inequality problems. We devote this paper to developing and improving the self-adaptive methods for solving the split feasibility problem. A new improved self-adaptive method is introduced for solving the split feasibility problem. As a special case, the minimum norm solution of the split feasibility problem can be approached iteratively. MSC: 47J25; 47J20; 49N45; 65J15
\end{abstract}

Keywords: split feasibility problem; self-adaptive method; projection; minimization problem; minimum-norm

\section{Introduction}

As we know, the original split feasibility problem (SFP) was introduced firstly by Censor and Elfving [1], and has received much attention since its inception in 1994. This is due to its applications in signal processing and image reconstruction, with particular progress in intensity-modulated radiation therapy; please, see [2-6].

Since the SFP is a special case of the convex feasibility problem (CFP), which is to find a point in the nonempty intersection of finitely many closed and convex sets, we next briefly review some historic approaches which relate to the CFP. The CFP is an important problem because many real-world inversion or estimation problems in engineering as well as in mathematics can be cast into this framework; see, e.g., Combettes [7], Bauschke and Borwein [8] and Kiwiel [9]. Traditionally, iterative projection methods for solving the CFP employ orthogonal projections onto convex sets (i.e., nearest point projections with respect to the Euclidean distance function); see, e.g., [10-14]. Much work has been done with generalized distance functions and the generalized projections associated with them suggested by Bregman [15].

In 1994, Censor and Elfving [1] investigated the use of different kinds of generalized projections in a single iterative process for solving the SFP. Their proposal is an iterative algorithm, which involves the computation of the inverse of a matrix, which is known to be a difficult task. That is why Byrne $[16,17]$ proposed the so-called $C Q$ algorithm, which generates a sequence by a recursive procedure with suitable step-size. The $C Q$ algorithm only involves the computations of the projections onto the sets $C$ and $Q$, respectively, and is therefore implementable in the case where these projections have closed-form expressions (e.g., $C$ and $Q$ are the closed balls or half-spaces). There is a large number of references on the CQ method in the literature; see, for instance, [18-34]. However, we have to

(c) 2013 Yao et al.: licensee Springer. This is an Open Access article distributed under the terms of the Creative Commons Attribution License (http://creativecommons.org/licenses/by/2.0), which permits unrestricted use, distribution, and reproduction in any medium, provided the original work is properly cited. 
remark that the determination of the step-size depends on the operator (matrix) norm (or the dominant eigenvalue of a matrix product). This means that in order to implement the $C Q$ algorithm, one has first to compute (or, at least, to estimate) the matrix norm of an operator, which is in general not an easy work in practice.

To overcome the above difficulty, the so-called self-adaptive method which permits stepsize being selected self-adaptively was developed. Note that this method is the application of the projection method of Goldstein [35], Levitin and Polyak [36] to a suitable variational inequality problem, which is among the simplest numerical methods for solving variational inequality problems. Nevertheless, the efficiency of this projection method depends strongly on the choice of the step-size parameter. If one chooses a small parameter such that it guarantees the convergence of the iterative sequence, the recursion leads to slow speed of convergence. On the other hand, if one chooses a large step-size to improve the speed of convergence, the generated sequence may not converge. In real applications to solving variational inequality problems, the Lipschitz constant may be difficult to estimate, even if the underlying mapping is linear, the case such as the SFP. Some self-adaptive methods for solving variational inequality problems have been developed according to the original Goldstein-Levitin-Polyak method [35, 36]. See, e.g., [37-45].

Motivated by the self-adaptive strategy, Zhang et al. [45] proposed their method by using variable step-sizes instead of the fixed step-sizes as in Censor et al. [46]. Also, a selfadaptive projection method was introduced by Zhao and Yang [29], and it was adopted by using the Armijo-like searches. The advantage of these algorithms lies in the fact that neither prior information about the matrix norm $A$ nor any other conditions on $Q$ and $A$ are required, and still convergence is guaranteed.

In this paper, we further develop and improve the self-adaptive methods for solving the SFP. An improved self-adaptive method is introduced for solving the SFP. As a special case, the minimum norm solution of the SFP can be approached iteratively.

\section{Framework and preliminary results}

Let $H_{1}$ and $H_{2}$ be two Hilbert spaces, and let $C$ and $Q$ be two closed and convex subsets of $H_{1}$ and $H_{2}$, respectively. Let $A: H_{1} \rightarrow H_{2}$ be a bounded linear operator. The split feasibility problem (SFP) is to find a point $x^{*}$ such that

$$
x^{*} \in C \text { and } A x^{*} \in Q \text {. }
$$

Next, we use $\Gamma$ to denote the solution set of the SFP, i.e., $\Gamma=\{x \in C: A x \in Q\}$.

In 1994, Censor and Elfving [1] investigated the use of different kinds of generalized projections in a single iterative process for solving the SFP. They were the first to propose the following algorithm which involved the computation of the inverse $A^{-1}$ :

$$
x_{k+1}=A^{-1} P_{Q}\left(P_{A(C)}\left(A x_{k}\right)\right), \quad k \geq 0,
$$

where $C$ and $Q$ are closed and convex sets in $\mathbb{R}^{n}$, while $A$ is a full rank $n \times n$ matrix and $A(C)=\left\{y \in \mathbb{R}^{n} \mid y=A x, x \in C\right\}$. Note that $A^{-1}$ is not easily executed. Consequently, Byrne $[16,17]$ proposed the so-called $C Q$ algorithm which generates a sequence $\left\{x_{n}\right\}$ by the recursive procedure

$$
x_{n+1}=P_{C}\left(x_{n}-\tau_{n} A^{*}\left(I-P_{Q}\right) A x_{n}\right),
$$


where the step-size $\tau_{n}$ is chosen in the interval $\left(0,2 /\|A\|^{2}\right)$. It is remarkable that the $C Q$ algorithm only involves the computations of the projections $P_{C}$ and $P_{Q}$ onto the sets $C$ and $Q$, respectively, and is therefore implementable in the case where $P_{C}$ and $P_{Q}$ have closedform expressions (e.g., $C$ and $Q$ are the closed balls or half-spaces). However, we observe that the determination of the step-size $\tau_{n}$ depends on the operator (matrix) norm $\|A\|$ (or the largest eigenvalue of $A^{*} A$ ). This means that for practical implementation of the $C Q$ algorithm, one has first to compute (or, at least, to estimate) the matrix norm of $A$, which is in general not an easy task in practice.

To overcome the above difficulty, the so-called self-adaptive method which permits stepsize $\tau_{n}$ being selected self-adaptively was developed. If we set

$$
f(x):=\frac{1}{2}\left\|A x-P_{Q} A x\right\|^{2},
$$

then the convex objective $f$ is differentiable and has a Lipschitz gradient given by

$$
\nabla f(x)=A^{*}\left(I-P_{Q}\right) A
$$

Thus, the $C Q$ algorithm (2) can be obtained by minimizing the following convex minimization problem

$$
\min _{x \in C} f(x)
$$

We know that a point $x^{*} \in C$ is a stationary point of problem (3) if it satisfies

$$
\left\langle\nabla f\left(x^{*}\right), x-x^{*}\right\rangle \geq 0, \quad \forall x \in C
$$

Thus, we can use a gradient projection algorithm below to solve the (SFP)

$$
x_{n+1}=P_{C}\left(x_{n}-\tau_{n} \nabla f\left(x_{n}\right)\right) \text {, }
$$

where $\tau_{n}$, the step-size at iteration $n$, is chosen in the interval $(0,2 / L)$, where $L$ is the Lipschitz constant of $\nabla f$.

The above method (5) has to be thought of as the application of the projection method of Goldstein [35], Levitin and Polyak [36] to the variational inequality problem (4), which is among the simplest numerical methods for solving variational inequality problems. Nevertheless, the efficiency of this projection method depends greatly on the choice of the parameter $\tau_{n}$. A small $\tau_{n}$ guarantees the convergence of the iterative sequence, but the recursion leads to slow speed of convergence. On the other hand, a large step-size will improve the speed of convergence, but the generated sequence may not converge. In real applications for solving variational inequality problems, the Lipschitz constant may be difficult to estimate, even if the underlying mapping is linear, the case such as the SFP.

The methods in Zhang et al. [45] and Censor et al. [46] were proposed for solving the multiple-sets split feasibility problem.

Algorithm 2.1 S1. Given a nonnegative sequence $\tau_{n}$ such that $\sum_{n=0}^{\infty} \tau_{n}<\infty, \delta \in(0,1)$, $\mu \in(0,1), \rho \in(0,1), \epsilon>0, \beta_{0}>0$, and arbitrary initial point $x_{0}$. Set $\gamma_{0}=\beta_{0}$ and $n=0$. 
S2. Find the smallest nonnegative integer $l_{n}$ such that $\beta_{n+1}=\mu^{l_{k}} \gamma_{k}$ and

$$
x_{n+1}=P_{C}\left(x_{n}-\beta_{n+1} \nabla f\left(x_{n}\right)\right) \text {, }
$$

which satisfies

$$
\beta_{n+1}\left\|\nabla f\left(x_{n}\right)-\nabla f\left(x_{n+1}\right)\right\|^{2} \leq(2-\delta)\left\langle x_{n}-x_{n+1}, \nabla f\left(x_{n}\right)-\nabla f\left(x_{n+1}\right)\right\rangle .
$$

S3. If

$$
\beta_{n+1}\left\|\nabla f\left(x_{n}\right)-\nabla f\left(x_{n+1}\right)\right\|^{2} \leq \rho\left(x_{n}-x_{n+1}, \nabla f\left(x_{n}\right)-\nabla f\left(x_{n+1}\right)\right),
$$

then set $\gamma_{n+1}=\left(1+\tau_{n+1}\right) \beta_{n+1}$; otherwise, set $\gamma_{n+1}=\beta_{n+1}$.

S4. If $\left\|e\left(x_{n}, \beta_{n}\right)\right\| \leq \epsilon$, stop; otherwise, set $n:=n+1$ and go to S2.

The following self-adaptive projection method was introduced by Zhao and Yang [29], and it was adopted by using the Armijo-like searches.

Algorithm 2.2 Given constants $\beta>0, \sigma \in(0,1)$ and $\gamma \in(0,1)$. Let $x_{0}$ be arbitrary. For $n=0,1, \ldots$, calculate

$$
x_{n+1}=P_{C}\left(x_{n}-\tau_{n} \nabla f\left(x_{n}\right)\right),
$$

where $\tau_{n}=\beta \gamma^{l_{n}}$ and $l_{n}$ is the smallest nonnegative integer $l$ such that

$$
f\left(P_{C}\left(x_{n}-\beta \gamma^{l} \nabla f\left(x_{n}\right)\right)\right) \leq f\left(x_{n}\right)-\sigma\left\langle\nabla f\left(x_{n}\right), x_{n}-P_{C}\left(x_{n}-\beta \gamma^{l} \nabla f\left(x_{n}\right)\right)\right\rangle .
$$

The advantage of Algorithm 2.1 and Algorithm 2.2 lies in the fact that neither prior information about the matrix norm $A$ nor any other conditions on $Q$ and $A$ are required, and still convergence is guaranteed.

We shall introduce our improved self-adaptive method for solving the SFP. In this respect, we need the ingredients introduced right now.

Let $C$ be a nonempty closed convex subset of a real Hilbert space $H$. A mapping $T: C \rightarrow$ $C$ is called nonexpansive if

$$
\|T x-T y\| \leq\|x-y\|, \quad \forall x, y \in C .
$$

A mapping $\psi: C \rightarrow C$ is said to be $\delta$-contractive if there exists a constant $\delta \in[0,1)$ such that

$$
\|\psi(x)-\psi(y)\| \leq \delta\|x-y\|, \quad \forall x, y \in C .
$$

Recall that the (nearest point or metric) projection from $H$ onto $C$, denoted by $P_{C}$, assigns to each $x \in H$ the unique point $P_{C}(x) \in C$ with the property

$$
\left\|x-P_{C}(x)\right\|=\inf \{\|x-y\|: y \in C\} .
$$

It is well known that the metric projection $P_{C}$ of $H$ onto $C$ has the following basic properties: 
(a) $\left\|P_{C}(x)-P_{C}(y)\right\| \leq\|x-y\|$ for all $x, y \in H$;

(b) $\left\langle x-y, P_{C}(x)-P_{C}(y)\right\rangle \geq\left\|P_{C}(x)-P_{C}(y)\right\|^{2}$ for every $x, y \in H$;

(c) $\left\langle x-P_{C}(x), y-P_{C}(x)\right\rangle \leq 0$ for all $x \in H$ and $y \in C$.

Next we adopt the following notation:

- $x_{n} \rightarrow x$ means that $x_{n}$ converges strongly to $x$;

- $x_{n} \rightarrow x$ means that $x_{n}$ converges weakly to $x$;

- $\omega_{w}\left(x_{n}\right):=\left\{x: \exists x_{n_{j}}-x\right\}$ is the weak $\omega$-limit set of the sequence $\left\{x_{n}\right\}$.

Recall that a function $f: H \rightarrow \mathbb{R}$ is called convex if

$$
f(\lambda x+(1-\lambda) y) \leq \lambda f(x)+(1-\lambda) f(y), \quad \forall \lambda \in(0,1), \forall x, y \in H .
$$

It is known that a differentiable function $f$ is convex if and only if the following relation holds:

$$
f(z) \geq f(x)+\langle\nabla f(x), z-x\rangle, \quad \forall z \in H .
$$

Recall that an element $g \in H$ is said to be a subgradient of $f: H \rightarrow \mathbb{R}$ at $x$ if

$$
f(z) \geq f(x)+\langle g, z-x\rangle, \quad \forall z \in H .
$$

If the function $f: H \rightarrow \mathbb{R}$ has at least one subgradient at $x$, it is said to be subdifferentiable at $x$. The set of subgradients of $f$ at the point $x$ is called the subdifferential of $f$ at $x$, and is denoted by $\partial f(x)$. A function $f$ is called subdifferentiable if it is subdifferentiable at all $x \in H$. If $f$ is convex and differentiable, then its gradient and subgradient coincide. A function $f: H \rightarrow \mathbb{R}$ is said to be weakly lower semi-continuous (w-lsc) at $x$ if $x_{n} \rightarrow x$ implies

$$
f(x) \leq \liminf _{n \rightarrow \infty} f\left(x_{n}\right) .
$$

$f$ is said to be w-lsc on $H$ if it is w-lsc at every point $x \in H$.

The first lemma is easy to prove.

Lemma 2.1 [14] Let $f(x):=\frac{1}{2}\left\|A x-P_{Q} A x\right\|^{2}$. Then

(i) $f$ is convex and differentiable;

(ii) $f$ is w-lsc on $C$.

Lemma 2.2 [47] Given $x^{*} \in H_{1}$. Then $x^{*}$ solves the SFP if and only if $x^{*}$ solves the fixed point equation

$$
x^{*}=P_{C}\left(x^{*}-\gamma A^{*}\left(I-P_{Q}\right) A x^{*}\right),
$$

where $\gamma>0$.

Lemma 2.3 [48] Assume that $\left\{a_{n}\right\}$ is a sequence of nonnegative real numbers such that

$$
a_{n+1} \leq\left(1-\gamma_{n}\right) a_{n}+\delta_{n},
$$

where $\left\{\gamma_{n}\right\}$ is a sequence in $(0,1)$ and $\left\{\delta_{n}\right\}$ is a sequence such that 
(1) $\sum_{n=1}^{\infty} \gamma_{n}=\infty$;

(2) $\lim \sup _{n \rightarrow \infty} \frac{\delta_{n}}{\gamma_{n}} \leq 0$ or $\sum_{n=1}^{\infty}\left|\delta_{n}\right|<\infty$.

Then $\lim _{n \rightarrow \infty} a_{n}=0$.

Lemma 2.4 [49] Let $\left\{s_{n}\right\}$ be a sequence of real numbers that does not decrease at infinity, in the sense that there exists a subsequence $\left\{s_{n_{i}}\right\}$ of $\left\{s_{n}\right\}$ such that $s_{n_{i}} \leq s_{n_{i}+1}$ for all $i \geq 0$. For every $n \geq n_{0}$, define an integer sequence $\{\tau(n)\}$ as

$$
\tau(n)=\max \left\{k \leq n: s_{n_{i}}<s_{n_{i}+1}\right\} .
$$

Then $\tau(n) \rightarrow \infty$ as $n \rightarrow \infty$ and for all $n \geq n_{0}$

$$
\max \left\{s_{\tau(n)}, s_{n}\right\} \leq s_{\tau(n)+1} .
$$

\section{Main results}

In this section we state and prove our main results.

Let $C$ and $Q$ be nonempty closed convex subsets of real Hilbert spaces $H_{1}$ and $H_{2}$, respectively. Let $\psi: C \rightarrow H_{1}$ be a $\delta$-contraction with $\delta \in\left[0, \frac{\sqrt{2}}{2}\right.$ ). Let $A: H_{1} \rightarrow H_{2}$ be a bounded linear operator.

Algorithm 3.1 For given $x_{0} \in C$, assume that $\left\{x_{n}\right\}$ has been constructed. If $\nabla f\left(x_{n}\right)=0$, then stop and $x_{n}$ is a solution of SFP (1). Otherwise, continue and compute $x_{n+1}$ by the recursion

$$
x_{n+1}=P_{C}\left[\alpha_{n} \psi\left(x_{n}\right)+\left(1-\alpha_{n}\right)\left(x_{n}-\frac{\rho_{n} f\left(x_{n}\right)}{\left\|\nabla f\left(x_{n}\right)\right\|^{2}} \nabla f\left(x_{n}\right)\right)\right], \quad n \geq 0,
$$

where $\left\{\alpha_{n}\right\} \subset(0,1)$ and $\left\{\rho_{n}\right\} \subset(0,2)$.

Theorem 3.1 Suppose that the SFP is consistent, that is, $\Gamma \neq \varnothing$. Assume that the following conditions hold:

(a) $\lim _{n \rightarrow \infty} \alpha_{n}=0$ and $\sum_{n=1}^{\infty} \alpha_{n}=\infty$;

(b) $\inf _{n} \rho_{n}\left(2-\rho_{n}\right)>0$.

Then $\left\{x_{n}\right\}$ defined by (6) converges strongly to $z$, which solves the following variational inequality:

$$
z \in \Gamma \quad \text { such that } \quad\langle z-\psi(z), z-x\rangle \leq 0 \quad \text { for all } x \in \Gamma .
$$

Proof First, it is obvious that the solution of the variational inequality (7) is unique (by the strong monotonicity of $I-\psi$ according to the related results in variational inequality), denoted by $z$. Then $z=P_{\Gamma}(\psi(z))$. We may assume that the sequence $\left\{x_{n}\right\}$ is infinite, that is, Algorithm 3.1 does not terminate in a finite number of iterations. Thus, $\nabla f\left(x_{n}\right) \neq 0$ for all $n$. From (6), we have

$$
\begin{aligned}
\left\|x_{n+1}-z\right\|^{2} & =\left\|P_{C}\left[\alpha_{n} \psi\left(x_{n}\right)+\left(1-\alpha_{n}\right)\left(x_{n}-\frac{\rho_{n} f\left(x_{n}\right)}{\left\|\nabla f\left(x_{n}\right)\right\|^{2}} \nabla f\left(x_{n}\right)\right)\right]-z\right\|^{2} \\
& \leq\left\|\alpha_{n}\left(\psi\left(x_{n}\right)-z\right)+\left(1-\alpha_{n}\right)\left(x_{n}-\frac{\rho_{n} f\left(x_{n}\right)}{\left\|\nabla f\left(x_{n}\right)\right\|^{2}} \nabla f\left(x_{n}\right)-z\right)\right\|^{2}
\end{aligned}
$$




$$
\begin{aligned}
\leq & \alpha_{n}\left\|\psi\left(x_{n}\right)-z\right\|^{2}+\left(1-\alpha_{n}\right)\left\|x_{n}-\frac{\rho_{n} f\left(x_{n}\right)}{\left\|\nabla f\left(x_{n}\right)\right\|^{2}} \nabla f\left(x_{n}\right)-z\right\|^{2} \\
\leq & \left(1-\alpha_{n}\right)\left[\left\|x_{n}-z\right\|^{2}+\frac{\rho_{n}^{2} f^{2}\left(x_{n}\right)}{\left\|\nabla f\left(x_{n}\right)\right\|^{2}}-\frac{2 \rho_{n} f\left(x_{n}\right)}{\left\|\nabla f\left(x_{n}\right)\right\|^{2}}\left\langle\nabla f\left(x_{n}\right), x_{n}-z\right\rangle\right] \\
& +\alpha_{n}\left(\left\|\psi\left(x_{n}\right)-\psi(z)\right\|+\|\psi(z)-z\|\right)^{2} .
\end{aligned}
$$

By the convexity of $f$ (Lemma 2.1) and the fact that $\nabla f(z)=0$ for $z \in \Gamma$, we deduce that

$$
f\left(x_{n}\right)=f\left(x_{n}\right)-f(z) \leq\left\langle\nabla f\left(x_{n}\right), x_{n}-z\right\rangle .
$$

Using the inequality $(a+b)^{2} \leq 2\left(a^{2}+b^{2}\right)$ for all $a, b \in \mathbb{R}$, we have

$$
\begin{aligned}
\left(\left\|\psi\left(x_{n}\right)-\psi(z)\right\|+\|\psi(z)-z\|\right)^{2} & \leq 2\left\|\psi\left(x_{n}\right)-\psi(z)\right\|^{2}+2\|\psi(z)-z\|^{2} \\
& \leq 2 \delta^{2}\left\|x_{n}-z\right\|^{2}+2\|\psi(z)-z\|^{2}
\end{aligned}
$$

From (8)-(10), we get

$$
\begin{aligned}
\left\|x_{n+1}-z\right\|^{2} \leq & \left(1-\alpha_{n}\right)\left[\left\|x_{n}-z\right\|^{2}-\rho_{n}\left(2-\rho_{n}\right) \frac{f^{2}\left(x_{n}\right)}{\left\|\nabla f\left(x_{n}\right)\right\|^{2}}\right] \\
& +2 \delta^{2} \alpha_{n}\left\|x_{n}-z\right\|^{2}+2 \alpha_{n}\|\psi(z)-z\|^{2} \\
\leq & {\left[1-\left(1-2 \delta^{2}\right) \alpha_{n}\right]\left\|x_{n}-z\right\|^{2}+\left(1-2 \delta^{2}\right) \alpha_{n} \frac{2\|\psi(z)-z\|^{2}}{1-2 \delta^{2}} } \\
\leq & \max \left\{\left\|x_{n}-z\right\|^{2}, \frac{2\|\psi(z)-z\|^{2}}{1-2 \delta^{2}}\right\} .
\end{aligned}
$$

By induction, we deduce

$$
\left\|x_{n+1}-z\right\|^{2} \leq \max \left\{\left\|x_{0}-z\right\|^{2}, \frac{2\|\psi(z)-z\|^{2}}{1-2 \delta^{2}}\right\}
$$

Hence, $\left\{x_{n}\right\}$ is bounded.

By using the firm nonexpansivity of $P_{C}$, we derive that

$$
\begin{aligned}
\left\|x_{n+1}-z\right\|^{2}= & \left\|P_{C}\left[\alpha_{n} \psi\left(x_{n}\right)+\left(1-\alpha_{n}\right)\left(x_{n}-\frac{\rho_{n} f\left(x_{n}\right)}{\left\|\nabla f\left(x_{n}\right)\right\|^{2}} \nabla f\left(x_{n}\right)\right)\right]-P_{C} z\right\|^{2} \\
\leq & \alpha_{n}\left\langle\psi\left(x_{n}\right)-z, x_{n+1}-z\right\rangle+\left(1-\alpha_{n}\right)\left(x_{n}-\frac{\rho_{n} f\left(x_{n}\right)}{\left\|\nabla f\left(x_{n}\right)\right\|^{2}} \nabla f\left(x_{n}\right)-z, x_{n+1}-z\right\rangle \\
= & \alpha_{n}\left\langle\psi\left(x_{n}\right)-\psi(z), x_{n+1}-z\right\rangle+\alpha_{n}\left\langle\psi(z)-z, x_{n+1}-z\right\rangle \\
& +\left(1-\alpha_{n}\right)\left\langle x_{n}-\frac{\rho_{n} f\left(x_{n}\right)}{\left\|\nabla f\left(x_{n}\right)\right\|^{2}} \nabla f\left(x_{n}\right)-z, x_{n+1}-z\right\rangle \\
\leq & \alpha_{n} \delta\left\|x_{n}-z\right\|\left\|x_{n+1}-z\right\|+\alpha_{n}\left\langle\psi(z)-z, x_{n+1}-z\right\rangle \\
& +\left(1-\alpha_{n}\right)\left\|x_{n}-\frac{\rho_{n} f\left(x_{n}\right)}{\left\|\nabla f\left(x_{n}\right)\right\|^{2}} \nabla f\left(x_{n}\right)-z\right\|\left\|x_{n+1}-z\right\| \\
= & \left(\alpha_{n} \delta\left\|x_{n}-z\right\|+\left(1-\alpha_{n}\right)\left\|x_{n}-\frac{\rho_{n} f\left(x_{n}\right)}{\left\|\nabla f\left(x_{n}\right)\right\|^{2}} \nabla f\left(x_{n}\right)-z\right\|\right)\left\|x_{n+1}-z\right\|
\end{aligned}
$$




$$
\begin{aligned}
& +\alpha_{n}\left\langle\psi(z)-z, x_{n+1}-z\right\rangle \\
\leq & \frac{1}{2}\left(\alpha_{n} \delta\left\|x_{n}-z\right\|+\left(1-\alpha_{n}\right)\left\|x_{n}-\frac{\rho_{n} f\left(x_{n}\right)}{\left\|\nabla f\left(x_{n}\right)\right\|^{2}} \nabla f\left(x_{n}\right)-z\right\|\right)^{2} \\
& +\frac{1}{2}\left\|x_{n+1}-z\right\|^{2}+\alpha_{n}\left\langle\psi(z)-z, x_{n+1}-z\right\rangle .
\end{aligned}
$$

It follows that

$$
\begin{aligned}
\left\|x_{n+1}-z\right\|^{2} \leq & \left(\alpha_{n} \delta\left\|x_{n}-z\right\|+\left(1-\alpha_{n}\right)\left\|x_{n}-\frac{\rho_{n} f\left(x_{n}\right)}{\left\|\nabla f\left(x_{n}\right)\right\|^{2}} \nabla f\left(x_{n}\right)-z\right\|\right)^{2} \\
& +2 \alpha_{n}\left\langle\psi(z)-z, x_{n+1}-z\right\rangle \\
\leq & \alpha_{n} \delta^{2}\left\|x_{n}-z\right\|^{2}+\left(1-\alpha_{n}\right)\left\|x_{n}-\frac{\rho_{n} f\left(x_{n}\right)}{\left\|\nabla f\left(x_{n}\right)\right\|^{2}} \nabla f\left(x_{n}\right)-z\right\|^{2} \\
& +2 \alpha_{n}\left\langle\psi(z)-z, x_{n+1}-z\right\rangle \\
\leq & \alpha_{n} \delta^{2}\left\|x_{n}-z\right\|^{2}+\left(1-\alpha_{n}\right)\left[\left\|x_{n}-z\right\|^{2}-\rho_{n}\left(2-\rho_{n}\right) \frac{f^{2}\left(x_{n}\right)}{\left\|\nabla f\left(x_{n}\right)\right\|^{2}}\right] \\
& +2 \alpha_{n}\left\langle\psi(z)-z, x_{n+1}-z\right\rangle \\
= & {\left[1-\left(1-\delta^{2}\right) \alpha_{n}\right]\left\|x_{n}-z\right\|^{2}+2 \alpha_{n}\left\langle\psi(z)-z, x_{n+1}-z\right\rangle } \\
& -\left(1-\alpha_{n}\right) \rho_{n}\left(2-\rho_{n}\right) \frac{f^{2}\left(x_{n}\right)}{\left\|\nabla f\left(x_{n}\right)\right\|^{2}} .
\end{aligned}
$$

Next, we will prove that $x_{n} \rightarrow z$ following the ideas in [49]. Set $s_{n}=\left\|x_{n}-z\right\|^{2}$ for all $n \geq 0$. Since $\alpha_{n} \rightarrow 0$ and $\inf _{n} \rho_{n}\left(2-\rho_{n}\right)>0$, we may assume, without loss of generality, that $\left(1-\alpha_{n}\right) \rho_{n}\left(2-\rho_{n}\right) \geq \sigma$ for some $\sigma>0$. Thus, we can rewrite (11) as

$$
s_{n+1}-s_{n}+\left(1-\delta^{2}\right) \alpha_{n} s_{n}+\frac{\sigma f^{2}\left(x_{n}\right)}{\left\|\nabla f\left(x_{n}\right)\right\|^{2}} \leq 2 \alpha_{n}\left\langle\psi(z)-z, x_{n+1}-z\right\rangle .
$$

Now, we consider two possible cases.

Case 1 . Assume that $\left\{s_{n}\right\}$ is eventually decreasing, i.e., there exists $N>0$ such that $\left\{s_{n}\right\}$ is decreasing for $n \geq N$. In this case, $\left\{s_{n}\right\}$ must be convergent, and from (12) it follows that

$$
\begin{aligned}
0 \leq \frac{\sigma f^{2}\left(x_{n}\right)}{\left\|\nabla f\left(x_{n}\right)\right\|^{2}} & \leq s_{n}-s_{n+1}-\left(1-\delta^{2}\right) \alpha_{n} s_{n}+2 \alpha_{n}\|\psi(z)-z\|\left\|x_{n+1}-z\right\| \\
& \leq s_{n}-s_{n+1}+M \alpha_{n}
\end{aligned}
$$

where $M>0$ is a constant such that $\sup _{n}\left\{2\|\psi(z)-z\|\left\|x_{n+1}-z\right\|\right\} \leq M$. Letting $n \rightarrow \infty$ in (13), we get

$$
\lim _{n \rightarrow \infty} f\left(x_{n}\right)=0
$$

Since $\left\{x_{n}\right\}$ is bounded, there exists a subsequence $\left\{x_{n_{k}}\right\}$ of $\left\{x_{n}\right\}$ converging weakly to $\tilde{x} \in C$.

From the weak lower semicontinuity of $f$, we have

$$
0 \leq f(\tilde{x}) \leq \liminf _{k \rightarrow \infty} f\left(x_{n_{k}}\right)=\lim _{n \rightarrow \infty} f\left(x_{n}\right)=0 .
$$


Hence, $f(\tilde{x})=0$, i.e., $A \tilde{x} \in Q$. This indicates that

$$
\omega_{w}\left(x_{n}\right) \subset \Gamma
$$

Furthermore, due to the property of the projection (c),

$$
\limsup _{n \rightarrow \infty}\left\langle\psi(z)-z, x_{n+1}-z\right\rangle=\max _{\omega \in \omega_{w}\left(x_{n}\right)}\left\langle\psi(z)-P_{\Gamma}(\psi(z)), \omega-P_{\Gamma}(\psi(z))\right\rangle \leq 0 .
$$

From (12), we obtain

$$
s_{n+1} \leq\left[1-\left(1-\delta^{2}\right) \alpha_{n}\right] s_{n}+2 \alpha_{n}\left\langle\psi(z)-z, x_{n+1}-z\right\rangle .
$$

Applying Lemma 2.3 to (14), we get $s_{n} \rightarrow 0$.

Case 2. Assume $\left\{s_{n}\right\}$ is not eventually decreasing. That is, there exists an integer $n_{0}$ such that $s_{n_{0}} \leq s_{n_{0}+1}$. Thus, we can define an integer sequence $\left\{\tau_{n}\right\}$ for all $n \geq n_{0}$ as follows:

$$
\tau(n)=\max \left\{k \in \mathbb{N} \mid n_{0} \leq k \leq n, s_{k} \leq s_{k+1}\right\} .
$$

Clearly, $\tau(n)$ is a non-decreasing sequence such that $\tau(n) \rightarrow+\infty$ as $n \rightarrow \infty$ and

$$
S_{\tau(n)} \leq S_{\tau(n)+1}
$$

for all $n \geq n_{0}$. In this case, we derive from (13) that

$$
\frac{\sigma f^{2}\left(x_{\tau(n)}\right)}{\left\|\nabla f\left(x_{\tau(n)}\right)\right\|^{2}} \leq M \alpha_{\tau(n)} \rightarrow 0
$$

It follows that

$$
\lim _{n \rightarrow \infty} f\left(x_{\tau(n)}\right)=0
$$

This implies that every weak cluster point of $\left\{x_{\tau(n)}\right\}$ is in the solution set $\Gamma$; i.e., $\omega_{w}\left(x_{\tau(n)}\right) \subset \Gamma$.

On the other hand, we note that

$$
\left\|x_{\tau(n)+1}-x_{\tau(n)}\right\| \leq \alpha_{\tau(n)}\left\|\psi\left(x_{\tau(n)}\right)-x_{\tau(n)}\right\|+\left(1-\alpha_{\tau(n)}\right) \frac{\rho_{\tau(n)} f\left(x_{\tau(n)}\right)}{\left\|\nabla f\left(x_{\tau(n)}\right)\right\|} \rightarrow 0,
$$

from which we can deduce that

$$
\begin{aligned}
\limsup _{n \rightarrow \infty}\left\langle\psi(z)-z, x_{\tau(n)+1}-z\right\rangle & =\limsup _{n \rightarrow \infty}\left\langle\psi(z)-z, x_{\tau(n)}-z\right\rangle \\
& =\max _{\omega \in \omega_{w}\left(x_{\tau(n)}\right)}\left\langle\psi(z)-P_{\Gamma}(\psi(z)), \omega-P_{\Gamma}(\psi(z))\right\rangle \\
& \leq 0 .
\end{aligned}
$$

Since $s_{\tau(n)} \leq s_{\tau(n)+1}$, we have from (12) that

$$
s_{\tau(n)} \leq \frac{2}{1-\delta^{2}}\left\langle\psi(z)-z, x_{\tau(n)+1}-z\right\rangle .
$$


Combining (15) and (16) yields

$$
\limsup _{n \rightarrow \infty} s_{\tau(n)} \leq 0
$$

and hence

$$
\lim _{n \rightarrow \infty} s_{\tau(n)}=0
$$

From (14), we have

$$
\limsup _{n \rightarrow \infty} s_{\tau(n)+1} \leq \limsup _{n \rightarrow \infty} s_{\tau(n)} .
$$

Thus,

$$
\lim _{n \rightarrow \infty} s_{\tau(n)+1}=0 .
$$

From Lemma 2.4, we have

$$
0 \leq s_{n} \leq \max \left\{s_{\tau(n)}, s_{\tau(n)+1}\right\}
$$

Therefore, $s_{n} \rightarrow 0$. That is, $x_{n} \rightarrow z$. This completes the proof.

From Theorem 3.1, we can deduce easily the following algorithm and corollary.

Algorithm 3.2 For given $x_{0} \in C$, assume that $\left\{x_{n}\right\}$ has been constructed. If $\nabla f\left(x_{n}\right)=0$, then stop and $x_{n}$ is a solution of SFP (1). Otherwise, continue and compute $x_{n+1}$ by the recursion

$$
x_{n+1}=P_{C}\left[\left(1-\alpha_{n}\right)\left(x_{n}-\frac{\rho_{n} f\left(x_{n}\right)}{\left\|\nabla f\left(x_{n}\right)\right\|^{2}} \nabla f\left(x_{n}\right)\right)\right], \quad n \geq 0,
$$

where $\left\{\alpha_{n}\right\} \subset(0,1)$ and $\left\{\rho_{n}\right\} \subset(0,2)$.

Theorem 3.2 Suppose that the SFP is consistent, that is, $\Gamma \neq \varnothing$. Assume that the following conditions hold:

(a) $\lim _{n \rightarrow \infty} \alpha_{n}=0$ and $\sum_{n=1}^{\infty} \alpha_{n}=\infty$;

(b) $\inf _{n} \rho_{n}\left(2-\rho_{n}\right)>0$.

Then $\left\{x_{n}\right\}$ defined by (17) converges strongly to the minimum norm solution of the SFP.

\section{Concluding remarks}

This work contains our study dedicated to developing and improving self-adaptive methods for solving the split feasibility problem. We have introduced our improved selfadaptive method for solving the split feasibility problem. As a special case, the minimum norm solution of the split feasibility problem can be approached iteratively. This study is motivated by relevant applications for solving many real-world problems, which give rise to mathematical models in the sphere of variational inequality problems. 


\section{Competing interests}

The authors declare that they have no competing interests.

\section{Authors' contributions}

The authors completed the paper together. All authors read and approved the final manuscript.

\section{Author details}

${ }^{1}$ Department of Mathematics, Tianjin Polytechnic University, Tianjin, 300387, China. ${ }^{2}$ Faculty of Applied Sciences, University 'Politehnica' of Bucharest, Splaiul Independentei 313, Bucharest, 060042, Romania. ${ }^{3}$ Department of Information Management, Cheng Shiu University, Kaohsiung, 833, Taiwan.

\section{Acknowledgements}

The first author was supported in part by NSFC 11071279 and NSFC 71161001-G0105. The third author was partially supported by NSC 101-2628-E-230-001-MY3

\section{Received: 21 May 2013 Accepted: 10 July 2013 Published: 25 July 2013}

\section{References}

1. Censor, Y, Elfving, T: A multiprojection algorithm using Bregman projections in a product space. Numer. Algorithms 8 , 221-239 (1994)

2. Censor, Y, Bortfeld, T, Martin, B, Trofimov, A: A unified approach for inversion problems in intensity-modulated radiation therapy. Phys. Med. Biol. 51, 2353-2365 (2006)

3. Stark, H (ed.): Image Recovery: Theory and Applications. Academic Press, San Diego (1987)

4. Ceng, LC, Ansari, QH, Yao, JC: An extragradient method for solving split feasibility and fixed point problems. Comput. Math. Appl. 64, 633-642 (2012)

5. Ceng, LC, Ansari, QH, Yao, JC: Mann type iterative methods for finding a common solution of split feasibility and fixed point problems. Positivity 16, 471-495 (2012)

6. Ceng, LC, Ansari, QH, Yao, JC: Relaxed extragradient methods for finding minimum-norm solutions of the split feasibility problem. Nonlinear Anal. 75, 2116-2125 (2012)

7. Combettes, PL: The foundations of set theoretic estimation. Proc. IEEE 81, 182-208 (1993)

8. Bauschke, HH, Borwein, JM: On projection algorithms for solving convex feasibility problems. SIAM Rev. 38, 367-426 (1996)

9. Kiwiel, KC: Block-iterative surrogate projection methods for convex feasibility problems. Technical report, Systems Research Inst., Polish Academy of Sciences, Newelska 6, 01-447, Warsaw, Poland (December 1992)

10. Butnariu, D, Censor, Y: On the behavior of a block-iterative projection method for solving convex feasibility problems. Int. J. Comput. Math. 34, 79-94 (1990)

11. Censor, Y: Row-action methods for huge and sparse systems and their applications. SIAM Rev. 23, 444-464 (1981)

12. Gubin, LG, Polyak, BT, Raik, EV: The method of projection for finding the common point of convex sets. U.S.S.R. Comput. Math. Math. Phys. 7, 1-24 (1967)

13. Han, SP: A successive projection method. Math. Program. 40, 1-14 (1988)

14. Iusem, AN, De Pierro, AR: On the convergence of Han's method for convex programming with quadratic objective. Math. Program. 52, 265-284 (1991)

15. Bregman, LM: The relaxation method of finding the common point of convex sets and its application to the solution of problems in convex programming. U.S.S.R. Comput. Math. Math. Phys. 7, 200-217 (1967)

16. Byrne, C: Iterative oblique projection onto convex subsets and the split feasibility problem. Inverse Probl. 18, 441-453 (2002)

17. Byrne, C: A unified treatment of some iterative algorithms in signal processing and image reconstruction. Inverse Probl. 20, 103-120 (2004)

18. Byrne, C: Bregman-Legendre multidistance projection algorithms for convex feasibility and optimization. In: Butnariu, D, Censor, Y, Reich, S (eds.) Inherently Parallel Algorithms in Feasibility and Optimization and Their Applications, pp. 87-100. Elsevier, Amsterdam (2001)

19. Censor, Y, Segal, A: The split common fixed point problem for directed operators. J. Convex Anal. 16, 587-600 (2009)

20. Dang, Y, Gao, Y: The strong convergence of a KM-CQ-like algorithm for a split feasibility problem. Inverse Probl. 27, Article ID 015007 (2011)

21. Fukushima, M: A relaxed projection method for variational inequalities. Math. Program. 35, 58-70 (1986)

22. Qu, B, Xiu, N: A note on the CQ algorithm for the split feasibility problem. Inverse Probl. 21, 1655-1665 (2005)

23. Wang, F, Xu, HK: Approximating curve and strong convergence of the CQ algorithm for the split feasibility problem. J. Inequal. Appl. (2010). doi:10.1155/2010/102085

24. Wang, F, Xu, HK: Cyclic algorithms for split feasibility problems in Hilbert spaces. Nonlinear Anal. 74, $4105-4111$ (2011)

25. Wang, Z, Yang, Q, Yang, Y: The relaxed inexact projection methods for the split feasibility problem. Appl. Math. Comput. (2010). doi:10.1016/j.amc.2010.11.058

26. Xu, HK: A variable Krasnosel'skii-Mann algorithm and the multiple-set split feasibility problem. Inverse Probl. 22 2021-2034 (2006)

27. Xu, HK: Averaged mappings and the gradient-projection algorithm. J. Optim. Theory Appl. 150, $360-378$ (2011)

28. Yang, Q, Zhao, J: Several solution methods for the split feasibility problem. Inverse Probl. 21, 1791-1799 (2005)

29. Zhao, J, Yang, Q: Self-adaptive projection methods for the multiple-sets split feasibility problem. Inverse Probl. 27, Article ID 035009 (2011)

30. Yao, Y, Wu, J, Liou, YC: Regularized methods for the split feasibility problem. Abstr. Appl. Anal. 2012, Article ID 140679 (2012)

31. Ceng, LC, Ansari, QH, Yao, JC: Extragradient-projection method for solving constrained convex minimization problems. Numer. Algebra Control Optim. 1, 341-359 (2011)

32. Ceng, LC, Ansari, QH, Yao, JC: Some iterative methods for finding fixed points and for solving constrained convex minimization problems. Nonlinear Anal. 74, 5286-5302 (2011) 
33. Ceng, LC, Ansari, QH, Wen, CF: Multi-step implicit iterative methods with regularization for minimization problems and fixed point problems. J. Inequal. Appl. 2013, 240 (2013). doi:10.1186/1029-242X-2013-240

34. Ceng, LC, Ansari, QH, Wen, CF: Implicit relaxed and hybrid methods with regularization for minimization problems and asymptotically strict pseudocontractive mappings in the intermediate sense. Abstr. Appl. Anal. 2013, Article ID 854297 (2013)

35. Goldstein, AA: Convex programming in Hilbert space. Bull. Am. Math. Soc. 70, 709-710 (1964)

36. Levitin, ES, Polyak, BT: Constrained minimization problems. U.S.S.R. Comput. Math. Math. Phys. 6, 1-50 (1966)

37. Han, D: Solving linear variational inequality problems by a self-adaptive projection method. Appl. Math. Comput. 182, 1765-1771 (2006)

38. Han, D: Inexact operator splitting methods with self-adaptive strategy for variational inequality problems. J. Optim. Theory Appl. 132, 227-243 (2007)

39. Han, D, Sun, W: A new modified Goldstein-Levitin-Polyak projection method for variational inequality problems. Comput. Math. Appl. 47, 1817-1825 (2004)

40. He, BS, He, X, Liu, H, Wu, T: Self-adaptive projection method for co-coercive variational inequalities. Eur. J. Oper. Res. $196,43-48(2009)$

41. He, BS, Yang, H, Meng, Q, Han, D: Modified Goldstein-Levitin-Polyak projection method for asymmetric strong monotone variational inequalities. J. Optim. Theory Appl. 112, 129-143 (2002)

42. He, BS, Yang, H, Wang, SL: Alternating direction method with self-adaptive penalty parameters for monotone variational inequalities. J. Optim. Theory Appl. 106, 337-356 (2000)

43. Liao, LZ, Wang, SL: A self-adaptive projection and contraction method for monotone symmetric linear variational inequalities. Comput. Math. Appl. 43, 41-48 (2002)

44. Yang, Q: On variable-step relaxed projection algorithm for variational inequalities. J. Math. Anal. Appl. 302, 166-179 (2005)

45. Zhang, W, Han, D, Li, Z: A self-adaptive projection method for solving the multiple-sets split feasibility problem. Inverse Probl. 25, Article ID 115001 (2009)

46. Censor, Y, Elfving, T, Kopf, N, Bortfeld, T: The multiple-sets split feasibility problem and its applications for inverse problems. Inverse Probl. 21, 2071-2084 (2005)

47. Xu, HK: Iterative methods for the split feasibility problem in infinite-dimensional Hilbert spaces. Inverse Probl. 26, Article ID 105018 (2010)

48. Xu, HK: Iterative algorithms for nonlinear operators. J. Lond. Math. Soc. 66, 240-256 (2002)

49. Mainge, PE: Strong convergence of projected subgradient methods for nonsmooth and nonstrictly convex minimization. Set-Valued Anal. 16, 899-912 (2008)

doi:10.1186/1687-1812-2013-201

Cite this article as: Yao et al.: Strong convergence of a self-adaptive method for the split feasibility problem. Fixed Point Theory and Applications 2013 2013:201.

\section{Submit your manuscript to a SpringerOpen ${ }^{\circ}$ journal and benefit from:}

- Convenient online submission

Rigorous peer review

- Immediate publication on acceptance

- Open access: articles freely available online

- High visibility within the field

- Retaining the copyright to your article 